\title{
One-Stage Decannulation Procedure for Patients Undergoing Oral and Oropharyngeal Oncological Surgeries and Prophylactic Tracheotomy
}

\author{
Oshri Wasserzug ${ }^{1}$, Nimrod Adi ${ }^{2}$, Oren Cavel $^{1}$, Noam Weizman ${ }^{1}$, Ahmad Safadi ${ }^{1}$, Joseph Vital ${ }^{1}$, \\ Patrick Sorkin ${ }^{2}$, Dan M. Fliss ${ }^{1}$ and Ziv Gil ${ }^{*}, 1,3,4$
}

${ }^{1}$ Department of Otolaryngology Head and Neck Surgery, ${ }^{2}$ Intensive Care Unit, and ${ }^{3}$ Skull Base Surgery Service, ${ }^{4}$ The Laboratory for Applied Cancer Research, Tel Aviv Sourasky Medical Center, Sackler Faculty of Medicine, Tel Aviv University, Tel Aviv, Israel

\begin{abstract}
Objective: Decannulation of patients with tracheotomy usually requires decrease in tracheostomy tube size, capping for 24-48 hours and observation after tube removal. Delay in decannulation may increase cardiopulmonary load, prolong hospitalization and cause patient distress. We propose a one-stage procedure in an intensive care unit (ICU) setting for patients undergoing head and neck surgeries and temporary tracheotomy.

Study Design and Setting: Patients undergoing resection of head and neck tumors involving the oral cavity or oropharynx in a tertiary cancer center were prospectively studied. Following clinical and laboratory assessments, the tracheostomy tube was removed under cardiopulmonary monitoring in the ICU.

Results: All 24 study patients underwent successful decannulation and were discharged 24 hours later. Follow-up time was 5 months. None of them required reintubation or recannulation.

Conclusion: A one-stage decannulation is feasible and safe for patients undergoing resection of head and neck tumors involving the oral cavity or oropharynx. This procedure may lessen hospitalization time and reduce patient's distress.
\end{abstract}

Keywords: Cancer, tracheostomy, oral cavity, airway, oncology.

\section{INTRODUCTION}

Patients undergoing head and neck tumor resection may require airway protection in the form of temporary tracheostomy following surgery. The tracheostomy tube is usually removed several days after the operation when edema and swelling in the upper airway have subsided. A common practice for decannulation is to gradually decrease the inner diameter (ID) of the tracheostomy tube from $8 \mathrm{~mm}$ to $4 \mathrm{~mm}[1,2]$. If tolerated, the tube is capped and removed when the patient no longer has reparatory distress or aspirations. Following decannulation, the patient is monitored for another 24-48 hours before discharge. The duration of the whole decannulation procedure is 96 hours. A short decannulation protocol used in some head and neck services is downsizing and capping of the tracheostomy tube on day 1, morning decannulation and observation on day 2 and discharge on day 3. Delay in decannulation increases the risk for aspirations, causes considerable distress to the patient and may delay his discharge. Furthermore, capping of the tracheostomy tube inevitably raises upper airway resistance, which may further increase the load on the patient's respiratory and cardiovascular systems and may cause cardiopulmonary distress in patients with prior respiratory or cardiac problems.

*Address correspondence to this author at the Department of Otolaryngology Head and Neck Surgery, Tel Aviv Sourasky Medical Center, 6 Weizmann Street, Tel Aviv, 64239, Israel; Tel: 972-3-6973544; Fax: 972-3-6973543; E-mail: ziv@baseofskull.org
To overcome these problems, we propose a one-stage decannulation procedure in the intensive care unit (ICU) for adult patients undergoing resection of head and neck tumors involving the oral cavity or oropharynx with temporary tracheostomy.

\section{MATERIALS AND METHODS}

From May 2006 to August 2008, all patients who underwent a tracheotomy for airway protection after oral cavity or oropharyngeal tumor resections were enrolled in this prospective study. All the patients had a Portex ${ }^{\circledR}$ (Smith Medical, Ashford, Kent, UK), cuffed tracheostomy tubes without window, with an inner diameter of 7.5 or $8 \mathrm{~mm}$. Only adult patients who were off ventilator and without need for respiratory support, had a normal fiber-optic examination of the upper airway, a leak around cuff, no dyspnea, stable arterial blood gases, stable hemodynamic status, no active infection and normal or adequate spirometry were included. Patients with chronic aspirations, upper airway obstruction, New-York class IV cardiac status, sepsis, bronchopulmonary infection or severe obstructive sleep apnea were excluded. The follow up period was 5 months after discharge. The study protocol was approved by the institutional review board committee.

\section{Decannulation Protocol}

The primary endpoint of our study was the decannulation period. Patients who fulfilled study entry criteria were admitted to the ICU where they underwent a comprehensive clinical and laboratory evaluation, including evaluation of 
blood pressure and heart rate, spirometry (maximal expiratory pressure of at least $40 \mathrm{cmH}_{2} \mathrm{O}$ ), arterial blood gases $\left(\mathrm{PaCO}_{2}<60 \mathrm{mmHg}\right)$ and body temperature. If all measures were within normal limits, the tracheostomy tube was removed and the stoma was loosely bandaged with gauze. The patient was then observed overnight for routine monitoring of electrocardiogram and blood $\mathrm{O}_{2}$ saturation. Within 24 hours after decannulation the patients were discharged from the ICU if arterial blood gases showed a $\mathrm{pH}$ $>7.35$ with $<5 \%$ increase in $\mathrm{PaCO}_{2}$. Fig. (1) displays the study protocol.

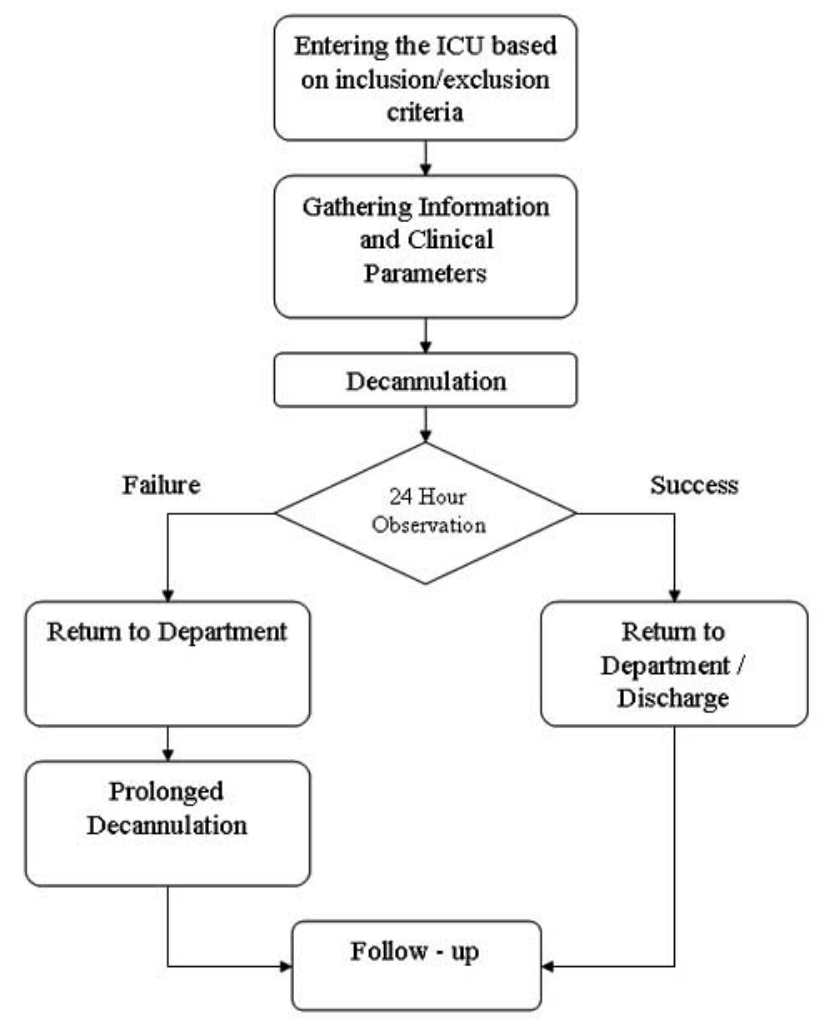

Fig. (1). Decannulation flowchart.

\section{RESULTS}

Twenty - four patients with oral cavity or oropharyngeal tumors underwent resection of the tumor with prophylactic tracheotomy. All of these 24 patients (mean age $49.4 \pm 14.9$ years, range 20-71, median 52, 12 males, 12 females) underwent a one-stage decannulation procedure in the ICU according to the protocol described above.

Table 1 summarizes their demographic and clinical characteristics. All the patients underwent successful decannulation on the same admission. The mean interval between surgery and decannulation was $6.59 \pm 1.6$ days (range 5-12, median 6). All the patients were discharged from the ICU within $<24$ hours of admission. They were discharged from the hospital 24 to 48 hours after decannulation. None of them had respiratory distress before discharge. They were all followed-up in an outpatient setting for 5 months after discharge, and none of them required recannulation or reintubation during the follow-up period.

\section{DISCUSSION}

The scientific literature on decannulation of patients with tracheostomy is limited, and the available publications relate mainly to the pediatric population [3-6]. To date, there is no evidence-based protocol for decannulation of patients after head and neck surgery. The main decannulation method practiced today is to gradually reduce the diameter of the tracheostomy tube $[1,2]$. It has been suggested that the effective diameter of the tracheostomy tube can be reduced by placing a cork in the lumen and gradually blocking it until total occlusion is tolerated [7]. Ceriana et al. proposed a protocol for weaning tracheotomized patients who are on long-term mechanical ventilation [2]. Those authors considered patients as being suitable if they were clinically stable, had a $\mathrm{PaCO} 2<60 \mathrm{mmHg}$, no delirium, no subglottic stenosis, and had proper swallowing function and a maximal expiratory pressure of at least $40 \mathrm{cmH} 2 \mathrm{O}$ (indicating the capability of removing secretions). If all those criteria were met, the tracheostomy tube was downsized to a $\leq 6 \mathrm{~mm}$ ID. The patient was then decannulated four days later if arterial blood gases showed a $\mathrm{pH}>7.35$ with $<5 \%$ increase in $\mathrm{PaCO}_{2}$. The failure rate in that study was $<3 \%$.

Decannulation protocols in the pediatric population relate mainly to children with tracheolaryngeal lesions or malformations in which the tracheostomy tube is in place for many months or years [8]. Subglottic stenosis, suprastomal granulations and primary tracheal lesions or malformations are frequent in such patients. That population clearly differs substantially from our cohort. We focused on a specific group of patients who underwent tracheotomy for airway protection after head and neck surgeries that involved the upper aerodigestive tracts. Our patients required airway protection for only a few days, until tissue swelling subsided. Throughout this time period patients were fed using a nasogastric tube or a percutaneous endoscopic gastrostomy (PEG).

In this prospective study, we demonstrated that decannulation can be performed safely as a one-stage procedure in the ICU without further evaluations. Alternatively, the observation could be performed in a step down unit to reduce the cost of ICU admission. The primary endpoint of our study was the decannulation period. Since our previous decannulation protocol required in at least 3-4 days (on day 1- decrease the diameter from $8 \mathrm{~mm}$ to $6 / 4$ $\mathrm{mm}$, on day 2- capping, on day 3- decannulation and monitored overnight), our new protocol reduced the decannulation period to less than 24 hours. We did not evaluate the impact of the protocol on hospitalization period which may be affected by other factors such as complications and availability of rehabilitation facilities.

Theoretically, tracheostomy tubes should decrease airflow resistance, but in fact this does not occur because of the small radius of the canulla $[9,10]$. The resistance to flow of air through the tube, represented by the Poiseuille equation, is inversely proportional to the radius of the tube raised to the $4^{\text {th }}$ power (when flow is laminar). When flow becomes turbulent (as in the case of a small tube diameter or capping), airways resistance becomes inversely proportional to the radius of the canulla raised to the $5^{\text {th }}$ power. Thus, 
Table 1. Demographic and Clinical Variables

\begin{tabular}{|c|c|c|c|}
\hline \multicolumn{2}{|c|}{ Variable } & \multirow{2}{*}{$\begin{array}{c}\text { No. of Patients } \\
24\end{array}$} & \multirow{2}{*}{$\begin{array}{c}\text { Percent } \\
100\end{array}$} \\
\hline Age, years & Range: $20-71$ (mean $=49.4 \pm 14.9$, median $52 \mathrm{yrs})$ & & \\
\hline \multirow{2}{*}{ Gender } & Male & 12 & 50 \\
\hline & Female & 12 & 50 \\
\hline \multirow{5}{*}{ Site of tumor } & Oral tongue & 11 & 45.9 \\
\hline & Floor of mouth & 5 & 20.8 \\
\hline & Mandible & 4 & 16.7 \\
\hline & Retromolar trigone & 2 & 8.3 \\
\hline & Buccal mucosa & 2 & 8.3 \\
\hline \multirow{4}{*}{ Extent of neck dissection } & SND (unilateral/bilateral) & $17(10 / 7)$ & 70.9 \\
\hline & MRND & 3 & 12.5 \\
\hline & RND & 3 & 12.5 \\
\hline & No neck dissection & 1 & 4.17 \\
\hline \multirow{4}{*}{ T stage } & 1 & 5 & 20.8 \\
\hline & 2 & 11 & 45.8 \\
\hline & 3 & 4 & 16.7 \\
\hline & 4 & 4 & 16.7 \\
\hline \multirow{4}{*}{$\mathrm{N}$ stage } & N0 & 9 & 37.5 \\
\hline & N1 & 4 & 16.7 \\
\hline & $\mathrm{N} 2 \mathrm{a} / \mathrm{b} / \mathrm{c}$ & 9 & 37.5 \\
\hline & N3 & 2 & 8.3 \\
\hline \multirow{4}{*}{ Overall stage } & I & 5 & 20.8 \\
\hline & II & 9 & 37.5 \\
\hline & III & 5 & 20.8 \\
\hline & IV & 5 & 20.8 \\
\hline \multirow{6}{*}{ Reconstruction } & RFFF & 10 & 41.7 \\
\hline & Fibular free flap & 5 & 20.8 \\
\hline & ALT & 4 & 16.7 \\
\hline & Pectoralis major & 2 & 8.3 \\
\hline & None & 1 & 4.17 \\
\hline & Local flap & 2 & 8.3 \\
\hline
\end{tabular}

ICU = Intensive Care Unit, SND = Selective neck dissection, MRND = Modified, Radical Neck dissection, RND = Radical Neck Dissection, RFFF= Radial Forearm Free-Flap $\mathrm{ALT}=$ Antero-Lateral Thigh.

small reductions in tube radius result in large increase in resistance [9]. Similarly, capping causes a sudden drop in tube diameter, inducing significant increase in airway resistance [11,12]. Jaeger et al. [13] and Pierson [14] found that small tube diameter and irregular tube walls (as with secretions or capping) increase the resistance and work of breeding (WOB) in patients with tracheostomy. In a laboratory study it was also demonstrated that airway resistance and WOB varied inversely with the inner diameter of the tracheostomy tube [14]. Under all conditions studied, imposed WOB was low with a high diameter tracheostomy tube, but increased significantly with decreasing the tube diameter. Gao et al. measured the upper-airway resistance in patients with tracheostomies in order to determine decannulation readiness [11]. They found that some patients, who cannot tolerate plugging the tracheostomy tube, may be ready for decannulation. This was attributed to the tube creating a significant obstruction and turbulent airflow. They suggested that removing the tube without capping may improve breathing in these patients permitting decannulation [11]. Although we are not aware of studies reporting an increase in cardiopulmonary events during standard decannulation process, our protocol suggests decannulation without increasing WOB in this subpopulation of patients.

Some authors suggested as an alternative to tracheostomy, to leave the trans-oral or trans-nasal endotracheal tube for 1-2 days and monitor the patients in the intensive care unit until the airway is patent (see discussion by Gil et al. [15]).

Our protocol improves the patients' discomfort since they can communicate earlier and swallow freely relatively soon after surgery [9]. Furthermore, shortening the duration of tracheostomy can reduce the risk of aspiration as well as decrease hospitalization time and, therefore, overall treatment cost. Furthermore, it eliminates the need for capping (i.e., increasing airway resistance), which may prevent respiratory distress in patients with lung or cardiac problems and low pulmonary reserves. Other complications associated with tracheostomy, such as bleeding, stenosis, infection and fistula formation can be prevented as well [2]. 
Our success rate of $100 \%$ with the proposed decannulation plan indicates that a one-stage decannulation procedure is safe in the population we chose to study. Further studies are needed to evaluate the utility of this protocol for other groups of patients, including those with prolonged intubation.

In conclusion, a one-stage decannulation procedure carried out in the ICU is both feasible and safe for patients undergoing resection of head and neck tumors involving the oral cavity or oropharynx with prophylactic tracheotomy.

\section{ACKNOWLEDGEMENTS}

Esther Eshkol, MA, institutional scientific copy editor, is thanked for editorial assistance. Supported partially by grants form the Israeli Cancer Association, America-Israel Binational Science Foundation and Israeli Science Foundation to Ziv Gil.

\section{REFERENCES}

[1] Godwin JE, Heffner JE. Special critical care considerations in tracheostomy management. Clin Chest Med 1991; 12: 573-83.

[2] Ceriana P, Carlucci A, Navalesi P, et al. Weaning from tracheotomy in long-term mechanically ventilated patients: feasibility of a decisional flowchart and clinical outcome. Intensive Care Med 2003; 29: 845-8.

[3] Sasaki CT, Gaudet PT, Peerless A. Tracheostomy decannulation. Am J Dis Child 1978; 132: 266-9.
[4] Willis R, Myer C, Miller R, et al. Tracheotomy decannulation in the pediatric patient. Laryngoscope 1987; 97: 764-5.

[5] Holborow C. Complications of tracheostomy in infancy and childhood with special reference to difficulty in decannulation. J Otolaryngol Soc Aust 1969; 2: 15-7.

[6] Diamant H, Kinnman J, Okmian L. Decannulation difficulties in tracheotomized children. Acta Otolaryngol Suppl 1960; 158: 39-42.

[7] Diamant H, Kinnman J, Okmian L. Decannulation in children. Laryngoscope 1961; 71: 404-14.

[8] Gray RF, Todd NW, Jacobs IN. Tracheostomy decannulation in children: approaches and techniques. Laryngoscope 1998; 108: 812.

[9] Epstein SK. Anatomy and physiology of tracheostomy. Respir Care 2005; 50: 476-82.

[10] Chadda K, Louis B, Benaissa L, et al. Physiological effects of decannulation in tracheostomized patients. Intensive Care Med 2002; 28: 1761-7.

[11] Gao C, Zhou L, Wei C, et al. The evaluation of physiologic decannulation readiness according to upper airway resistance measurement. Otolaryngol Head Neck Surg 2008; 139: 535-40.

[12] Haberthur C, Fabry B, Stocker R, et al. Additional inspiratory work of breathing imposed by tracheostomy tubes and non-ideal ventilator properties in critically ill patients. Intensive Care Med 1999; 25: 514-9.

[13] Jaeger JM, Littlewood KA, Durbin CG, Jr. The role of tracheostomy in weaning from mechanical ventilation. Respir Care 2002; 47: 469-80; discussion 81-2.

[14] Pierson DJ. Tracheostomy and weaning. Respir Care 2005; 50: 526-33.

[15] Gil Z, Cohen JT, Spektor S, Shlomi B, Fliss DM. Anterior skull base surgery without prophylactic airway diversion procedures. Otolaryngol - Head Neck Surg 2003; 128(5): 681-5. 